\title{
MiR-17 and miR-93 Promote Tumor Progression by Targeting P2I in Patients with Chordoma
}

\author{
Wei Dong ${ }^{1, *}$ \\ Jingwu $\mathrm{Li}^{2, *}$ \\ Xiaoliu Dong ${ }^{3}$ \\ Wenjian Shi \\ Yu Zhang ${ }^{4}$ \\ Yongliang Liu' \\ 'Department of Neurosurgery, Tangshan \\ People's Hospital, Tangshan, Hebei, \\ People's Republic of China; ${ }^{2}$ Department \\ of Tumor Surgery, Tangshan People's \\ Hospital, Tangshan, Hebei, People's \\ Republic of China; ${ }^{3}$ Department of \\ Neurology, Tangshan People's Hospital, \\ Tangshan, Hebei, People's Republic of \\ China; ${ }^{4}$ Department of Neurological \\ Intensive Care Unit, Tangshan People's \\ Hospital, Tangshan, Hebei, People's \\ Republic of China \\ *These authors contributed equally to \\ this work
}

Objective: MicroRNAs have been implicated in the progression of various cancers. However, the role of microRNAs in chordoma remains to be further elucidated. Here, we purposed to character the role of two microRNAs, miR-17 and miR-93, and their potential mechanisms in chordoma.

Methods: The expression and prognostic value of miR-17 and miR-93 were assessed by the quantitative real-time polymerase chain reaction, Kaplan-Meier survival curve, and Cox regression analysis. The effects of miR-17/93 mimics on chordoma cell proliferation, colony formation, and invasion were analyzed by CCK-8 assay, colony formation assay, and transwell assay. The downstream target of miR-17/93 was further explored via luciferase reporter assay.

Results: High expression of miR-17/93 was identified in chordoma tissues, and was associated with poor prognosis. Overexpression of miR-17/93 contributed to cell proliferation, colony formation, and invasion. Mechanistically, we demonstrated that miR-17/93 directly targeted p21 and decreased the expression of p21. Besides, the rescue assay further confirmed the essential role of the miR-17/93-p21 axis in chordoma.

Conclusion: Our results revealed the potential oncogenic effect of the miR-17/93 on chordoma progression, and suggested that the miR-17/93-p21 axis served as a promising therapeutic target in chordoma.

Keywords: microRNA, miR-17, miR-93, p21, chordoma

\section{Introduction}

Chordoma, identified as a rare and locally aggressive mesenchymal neoplasm, is developed from residual notochord tissues. ${ }^{1-3}$ Chordoma dominantly occurs at the midline skeleton axis, commonly skull base and sacrum with an incidence rate of 0.84 per million. ${ }^{3}$ Currently, the optimal treatment of chordoma is maximal surgical resection with optional proton/photon-beam radiotherapy for its nature of resistance to traditional radio/chemotherapy. ${ }^{2,4,5}$ However, due to the difficulty of radical resection and high locoregional recurrent rate, the chordoma patients harbor a dismal prognosis, especially for recurrent patients. ${ }^{3}$ Thus, a deeper exploration of chordoma's oncogenesis and more effective treatment options are needed to prolong patient survival in chordoma.

MicorRNAs (miRNAs), a cluster of small endogenous non-coding RNAs that

Correspondence: Yongliang Liu Department of Neurosurgery, Tangshan People's Hospital, No. 65 Shengli Road, Lunan District, Tangshan, 06300I, Hebei, People's Republic of China

Email s_c_2000@I63.com diffusely existed in various species including animals and plants, are generally composed of 18-23 nucleotides and post-transcriptionally regulated the target gene expression via binding to the mRNA. ${ }^{6-8}$ Studies involving the role of miRNAs have attracted prominent attention in recent decades and miRNAs have been recognized to 
play an essential role as carcinogen or tumor suppressors in multiple tumors, such as acute myeloid leukemia, gastric cancer, colorectal carcinoma, and prostate cancer. $^{9-12}$ Additionally, studies have demonstrated that miRNAs participate in various biological and tumor-associated processes, including cell growth, cell differentiation, invasion potential, angiogenesis, epithelial-mesenchymal transition and metastasis, and many aberrantly expressed miRNAs have shown the effective diagnostic value and prognostic performance in various cancers. ${ }^{13}$

Till now, the prognostic role and functions of miRNAs in chordoma are poorly understood. Recent studies have indicated that several miRNAs may contribute to oncogenesis and act as a valuable prognostic indicator in chordoma, such as miR-1, miR-1237-3p, miR-155. ${ }^{14-16}$ However, the role of miR-17 and miR-93 in chordoma remains unclear. MiR-17, encoded by miR-17-92 cluster, is highly expressed in tumors and involved in tumor progression, and high expression of which is associated with adverse outcome in T-cell lymphoblastic lymphoma and Burkitt lymphoma. ${ }^{17,18}$ For miR-93 encoded by miR106b-25 cluster, a paralog of miR-17-92 cluster, increasing researches have identified the oncogenetic role of miR-93 and high expression of miR-93 acts as a risk variable for survival in hepatocellular carcinoma and gastric cancer. $^{19,20}$ Additionally, miR-17 and miR-93 have been identified to directly target $\mathrm{p} 21$ in various cancers. ${ }^{21-24}$

In the current study, we investigated the mRNA expression of miR-17, miR-93, and p21 in chordoma, and their clinical impact, survival performance were further analyzed. We also explored the functions of miR17/93 and p21 in chordoma cells. Our findings identified that the miR-17/93-p21 axis act as a novel prognostic indicator and therapeutic target for chordoma.

\section{Materials and Methods}

\section{Tissue Samples}

After informed consent signature and ethics approval, fresh tumor tissues were collected from 30 patients who received surgical treatment at Tangshan People's Hospital between 2008 April and 2016 September. Patients with a prior history of radiotherapy/chemotherapy were excluded. All included patients were confirmed as chordoma by the pathologist, and 5 nucleus pulposus tissues were used as a control as the previous studies indicated. $^{25-27}$ All tissues were immediately stored in the liquid nitrogen until RNA extraction.
Of the 30 chordoma patients, 21 patients were male and 9 female patients. Ten patients were skull base chordoma and the remaining 20 patients harbor spinal chordoma (sacrum, 16 patients; thoracic or cervical vertebra, 4 patients), and the ages ranged from 26 to 71 years old. All patients were histopathologically confirmed as conventional types of chordoma. $^{2}$ The detailed clinical characteristics of chordoma patients are shown in Table 1 and Supplementary Table 1. The follow-up information from each patient was collected for survival analysis and the median follow-up time was 50 months (4-96 months). During the follow-up, 13 (43.3\%) patients died and the overall survival (OS) was defined as the interval from the first surgery to patient death.

\section{Cell Culture and Transfection}

Chordoma cell lines MUG-Chor1 (CRL-3219) and UMChor1 (CRL-3270) were acquired from American Type Culture Collection (ATCC), and the chordoma cells were cultured in IMDM (ATCC, USA) and RPMI-1640 (ATCC, USA) with a ratio of $4: 1$ containing $10 \%$ FBS (Gibco, USA) and 1\% penicillin/streptomycin (Invitrogen, USA). Cells were routinely incubated under $5 \% \mathrm{CO}_{2}, 37^{\circ} \mathrm{C}$ environment in a humidified incubator.

MiR-17 mimics, miR-93 mimics and miRNA mimics negative control (miR-NC) were purchased from Ribibio (Guangzhou, China). To overexpress p21, the pcDNA3.1-p21 overexpressed plasmid (p21) and control vector (Vector) were bought from Genepharma (Shanghai, China). $50 \mathrm{nM}$ miRNA mimics or $2 \mu \mathrm{g}$ of plasmid were transfected into MUG-Chor1 and UM-chor1 cells using Lipofectamine 3000 (Invitrogen, USA) according to the manufacturer's instruction. 48 hours after treatment, cells were gathered for RNA or protein extraction.

\section{RNA Extraction and Quantitative Real-Time Polymerase Chain Reaction (qRT-PCR)}

Expression of miR-17/93 and p21 in chordoma tissues and cell lines were evaluated by qRT-PCR. Total RNA was extracted using TRIzol reagent (Invitrogen, USA) based on the protocol and the RNA quantify and quality was further examined. 1st strand cDNA was acquired via a HiScript II cDNA kit (Vazyme, Nanjing, China). PCR was carried out via the ABI 7500 PCR system (Applied Biosystems, USA) using SYBR-Green PCR mix (Vazyme, China). U6 small nuclear RNA and GAPDH were used to normalize the expression of miR-17/93 and p21, respectively. Primers of 
Table I Correlation Between Expression of miR-17, miR-93 and P2I with Clinicopathological Features

\begin{tabular}{|c|c|c|c|c|c|c|c|c|c|c|}
\hline \multirow[t]{2}{*}{ Variables } & \multirow[t]{2}{*}{ Number } & \multicolumn{3}{|c|}{ miR-I7 Expression } & \multicolumn{3}{|c|}{ miR-93 Expression } & \multicolumn{3}{|c|}{ p2I Expression } \\
\hline & & High & Low & $P$ value & High & Low & $P$ value & High & Low & $P$ value \\
\hline Age (years) & & & & 0.060 & & & 0.263 & & & 0.710 \\
\hline$>50$ & 12 & 9 & 3 & & 8 & 4 & & 5 & 7 & \\
\hline$\leq 50$ & 18 & 6 & 12 & & 7 & 11 & & 10 & 8 & \\
\hline Sex & & & & 1.00 & & & 1.00 & & & 0.427 \\
\hline Male & 21 & 10 & 11 & & 11 & 10 & & 9 & 12 & \\
\hline Female & 9 & 5 & 4 & & 4 & 5 & & 6 & 3 & \\
\hline Tumor location & & & & 1.00 & & & 0.439 & & & 0.121 \\
\hline Skull base & 10 & 5 & 5 & & 4 & 6 & & 7 & 3 & \\
\hline Spine & 20 & 10 & 10 & & 11 & 9 & & 8 & 12 & \\
\hline Tumor size & & & & 0.456 & & & 0.456 & & & 0.264 \\
\hline$\leq 3 \mathrm{~cm}$ & 12 & 5 & 7 & & 5 & 7 & & 8 & 4 & \\
\hline$>3 \mathrm{~cm}$ & 18 & 10 & 8 & & 10 & 8 & & 7 & 11 & \\
\hline Primary tumor & & & & $0.035^{*}$ & & & 0.214 & & & 0.682 \\
\hline Yes & 22 & 8 & 14 & & 9 & 13 & & 12 & 10 & \\
\hline No & 8 & 7 & 1 & & 6 & 2 & & 3 & 5 & \\
\hline Total resection & & & & 1.00 & & & 0.651 & & & 0.651 \\
\hline Yes & 6 & 3 & 3 & & 2 & 4 & & 2 & 4 & \\
\hline No & 24 & 12 & 12 & & 13 & 11 & & 13 & II & \\
\hline
\end{tabular}

Note: *Indicates statistical significance.

miRNA were purchased from Ribibio company (Guangzhou, China) and the sequences for p21/GAPDH primers (BGI Genomics, Beijing, China) were as follows: p21, 5'TCCAGCGACCTTCCTCATCCA-3' and 5'-TCCATAGCC TCTACTGCCACCA; GAPDH， 5'-AGGTCGGAGTCA ACGGATTTG-3' and 5'-GTGATGGCATGGACTGTGGT$3^{\prime}$. The expression of the target gene was calculated as $2^{-\Delta \Delta C t}$.

\section{CCK-8 Assay}

Cell proliferation was evaluated by the CCK- 8 assay. MUGChor $1\left(3 \times 10^{3}\right.$ cells per well $)$ and UM-Chor $1\left(2 \times 10^{3}\right.$ cells per well) in medium without antibiotics were seeded into 96well plate, and chordoma cells were then transfected with miR-17/93 mimics or miR-NC, p21/control vector triplicate. At each point $(0,24,48,72 \mathrm{~h})$ after transfection, $10 \mu \mathrm{L}$ CCK-8 (Dojindo, Japan) was applied in wells, following the plates were incubated at $37^{\circ} \mathrm{C}$ for $2 \mathrm{~h}$. Cells' absorbances at $450 \mathrm{~nm}$ were assessed using the microplate reader.

\section{Colony Formation Assay}

Six hundred MUG-Chor 1 cells and $1 \times 10^{3}$ UM-Chor 1 cells transfected with different groups (miR-NC, miR-17/93 mimics, with/without vector or p21) were plated in the 6-well plates. After a two-week incubation, cells were fixed via $4 \%$ methanol and subsequently stained using $0.1 \%$ crystal violet for $10 \mathrm{~min}$.

\section{Invasion Assay}

Cell invasion activity of MUG-Chor1 and UM-chor1 was determined by transwell assay. $8 \mu \mathrm{m}$ 24-well transwell chamber (Corning, USA) with matrigel (BD, Biosciences, USA) was used according to the instruction. Briefly, transfected MUG-Chor1 $\left(5 \times 10^{4}\right.$ cells per well) and UM-Chor1 $\left(2 \times 10^{4}\right.$ cells per well) cells were plated onto the upper chamber with $100 \mu \mathrm{L}$ serum-free and antibiotic-free culture. For the bottom chamber, $500 \mu \mathrm{L}$ medium with $10 \%$ of FBS was applied. The transwell chamber was incubated for $48 \mathrm{~h}$ at $37^{\circ} \mathrm{C}$, and cells were fixed with methanol. After removing the cells on the upper chamber with the cotton swab, the cells on the inferior chamber were stained using crystal violet $(0.1 \%)$ for a quarter, photographed, and countered with a microscope (Zeiss, Germany).

\section{Western Blot Analysis}

Total protein from chordoma cells was regularly lysed with RIPA buffer (Beyotime, China), and the concentrations were 
quantified by BCA assay (Applygen, China). $30 \mu \mathrm{g}$ protein was separated by $10 \%$ SDS-PAGE and then moved to the PVDF member (Sigma-Aldrich, USA) according to standard instruction. The anti-p21 antibody (1:1000, ab109520) and GAPDH antibody (1:8000, ab128915) were purchased from Abcam (UK). A chemiluminescence assay from SigmaAldrich (USA) was used for signal detection.

\section{Luciferase Reporter Assay}

The renilla/firefly luciferase reporter plasmid (psiCHECK2 vector, Promega) with $3^{\prime}$-UTR wild or mutant types of $\mathrm{p} 21$, and miR-17/93 mimics or miR-NC were routinely transfected in chordoma cells according to the protocol. $48 \mathrm{~h}$ after transfection, cells were collected for assessment of firefly and renilla luciferase activities with the help of a dual-luciferase reporter assay system (Promega, Madison, USA). Then, ratios of firefly to renilla were calculated, and the average of three independent experiments was recognized as final luciferase activity.

\section{Statistical Analysis}

All statistical analyses were performed by SPSS 21.0 (IBM, NY, USA). The expression of miR-17/93 and p21 was separated into two subgroups (high level or low level) using the median value, respectively. The relationships between miR17, miR-93, p21, and clinical features were analyzed via the chi-square test. To find potential correlations, Pearson's correlation analysis was used. Student's $t$-test was applied to compare data between two subgroups and for data with multi groups, one-way ANOVA was applied for different analyses. Kaplan Meier analysis and Log rank test were used for OS analysis. Univariable and multivariable Cox regression analyses were performed for recognizing potential independent prognostic variables. A $p$-value of less than 0.05 was considered to be of statistical significance.

\section{Results}

\section{Upregulation of MiR-I7/93 and Downregulation of P2I Was Correlated with Poor Outcome in Chordoma Patients}

We first evaluated the expression of miR-17/93 and p21 in 30 chordoma tissues and 5 normal tissues using qRT-PCR and our finding indicated that the expression of miR-17 was notably increased in chordoma tissues, compared with that in nucleus pulposus $(p<0.01$, Figure $1 \mathrm{~A})$. Similarly, the level of miR-93 was also higher in chordoma tissues $(p<0.05$, Figure 1B). In contrast, the expression of $\mathrm{p} 21$ was lower in chordoma tissues $(p<0.01$, Figure 1C).

We then divided the 30 chordoma patients into high/ low expression groups using the median values of miR$17 / 93$ and p21 and further analyzed the relationships between their expressions with clinical parameters and survival in chordoma. We found that the expression of miR-17 was significantly correlated with primary/recurrent tumors $(p=0.035)$ (Table 1). Besides, there was a trend that miR-17 expression positively correlated with
A

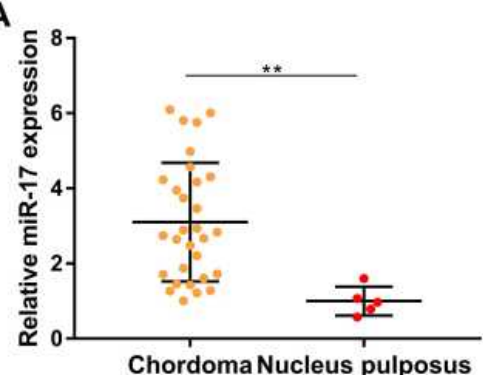

D

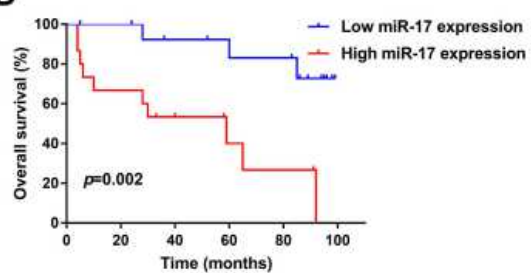

B

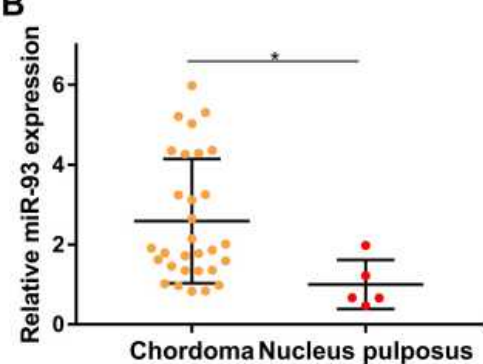

E

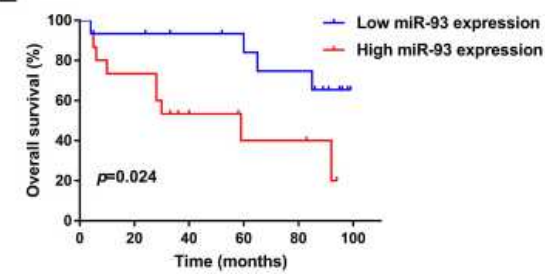

C

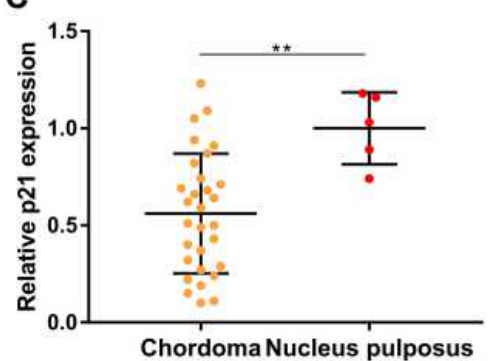

$\mathbf{F}$

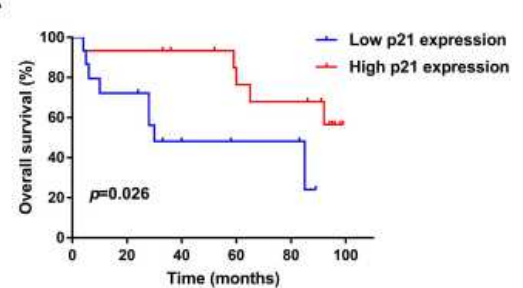

Figure I Prognostic value of miR-17, miR-93, and p2I in chordoma. (A-C) Expression of miR-17, miR-93, and p21 in chordoma tissues compared to nucleus pulposus tissues. (D-F) Kaplan-Meier survival analysis of miR-17, miR-93, and p2I in chordoma. $*_{p}<0.05$, ${ }^{*} p<0.01$. 
patient age $(p=0.060)$. However, no other correlations with clinical features including gender, location, tumor size, and types of resection were observed (Table 1). Kaplan Meier analysis indicated that patients with higher miR-17 expression had a shorter OS time than patients with lower miR-17 expression ( $p=0.002$, Figure 1D). Moreover, higher miR-93 expression was also associated with poor overall survival ( $p=0.024$, Figure $1 \mathrm{E}$ ), while patients with lower p21 expression harbored a poor outcome than patients with a higher p21 level $(p=0.026$, Figure 1F).

The univariate Cox analysis revealed that expression of miR-17 $(p=0.006)$, expression of miR-93 $(p=0.035), \mathrm{p} 21$ expression $(p=0.037)$, types of resection $(p=0.048)$, and tumor progression $(p<0.001)$ were associated with survival of chordoma patients, while other clinical parameters showed no prognostic value. More importantly, multivariate Cox analysis found that the expression of miR-17 $(p=0.048)$, tumor progression $(p<0.001)$ were independent predicted parameters for OS of chordoma patients (Table 2).

\section{MiR-I7 and MiR-93 Promoted the Malignant Behavior of Chordoma Cells}

Considering the prognostic roles of miR-17 and 93 in chordoma, we next investigated their potential function via overexpressing miR-17/93 in MUG-Chor1 and UM-Chor1 chordoma cells using miR-17/93 mimics. Using qRT-PCR, we confirmed the overexpression of miR-17/93 in miR-17/ 93 transfected cells (Figure 2A and B). CCK8 assay revealed that a remarked increase in cell viability of miR-17/93 group cells compared to the miR-NC cells (Figure 2C and D). Besides, colony formation assay measuring the cloning ability of MUG-Chor1 and UM-Chor1 cells revealed a significant increase of cloning cells in miR-17/93 transfected cells compared to the miR-NC group cells (Figure 2E and F). Moreover, invasion assay showed that invasion abilities of MUG-Chor1 and UM-Chor1 cells were increased after miR-17/93 overexpression (Figure $2 \mathrm{G}$ and $\mathrm{H}$ ). These data suggested that the oncogenic roles of miR-17 and miR93 in chordoma cells in vitro.

\section{MiR-I7 and MiR-93 Directly Targeted P2I in Chordoma Cells}

Bioinformatics analysis of starBase v2.0 and TargetScan showed p21 as a hypothetical target of miR-17 and miR-93 (Figure 3A), ${ }^{28}$ and previous research demonstrated that miR$17 / 93$ could function via directly targeting $\mathrm{p} 21 ;^{22,24}$ thus, we explored whether $\mathrm{p} 21$ as a target of miR-17/93 in chordoma. As shown in Figure 3B, overexpression of miR-17 or miR-93 could decrease the mRNA expression of p21 in chordoma cells. Accordingly, the protein expression of p21 was also decreased (Figure 3C). Moreover, luciferase reporter plasmids containing wide type $3^{\prime}$ UTR (p21-wt) and mutant 3' UTR (p21-mut) sequences of p21 were constructed, and chordoma cells were co-transfected with miR-17/93 mimics or miR-NC, and p21-wt or p21-mut plasmid. Co-transfection of miR-17/ miR-93 and p21-wt showed decreased luciferase activity, while no significant decrease in luciferase activity was observed when co-transfection of miR-17/miR-93 mimics and p21-mut (Figure 3D and E). Besides, there were significant negative correlations between miR-17 ( $\mathrm{r}=-0.49$, $p=0.007)$ or miR-93 ( $\mathrm{r}=-0.53, p=0.003)$ expression and mRNA level of p21 in chordoma tissues (Figure $3 \mathrm{~F}$ and $\mathrm{G}$ ).

Table 2 Univariate and Multivariate Cox Analysis of OS in Chordoma

\begin{tabular}{|c|c|c|c|c|}
\hline \multirow[t]{2}{*}{ Variables } & \multicolumn{2}{|c|}{ Univariate Analysis } & \multicolumn{2}{|c|}{ Multivariate Analysis } \\
\hline & HR (95\% Cl) & $P$ value & HR (95\% Cl) & $P$ value \\
\hline Age (>50 vs $\leq 50$ years) & $2.131(0.563-8.063)$ & 0.265 & & \\
\hline Sex (female vs male) & $0.850(0.260-2.785)$ & 0.789 & & \\
\hline Tumor location (cranial vs spinal) & $\mathrm{I} .13 \mathrm{I}(0.347-3.686)$ & 0.838 & & \\
\hline Tumor size $(>3$ vs $\leq 3 \mathrm{~cm})$ & $1.467(0.473-4.554)$ & 0.507 & & \\
\hline Primary tumor (yes vs no) & $0.665(0.183-2.423)$ & 0.537 & & \\
\hline Total resection (no vs yes) & $3.406(1.010-11.491)$ & $0.048^{*}$ & - & 0.110 \\
\hline Progression (yes vs no) & $9.549(3.482-26.183)$ & $<0.001 *$ & $6.850(2.451-19.145)$ & $<0.001 *$ \\
\hline miR-17 (high vs low) & $6.456(1.708-24.395)$ & $0.006 *$ & $5.097(1.014-25.611)$ & $0.048^{*}$ \\
\hline miR-93 (high vs low) & $3.690(1.097-12.419)$ & $0.035^{*}$ & - & 0.311 \\
\hline p2I expression (low vs high) & $3.723(1.08 \mid-12.825)$ & $0.037^{*}$ & - & 0.245 \\
\hline
\end{tabular}

Note: *Indicates statistical significance. 

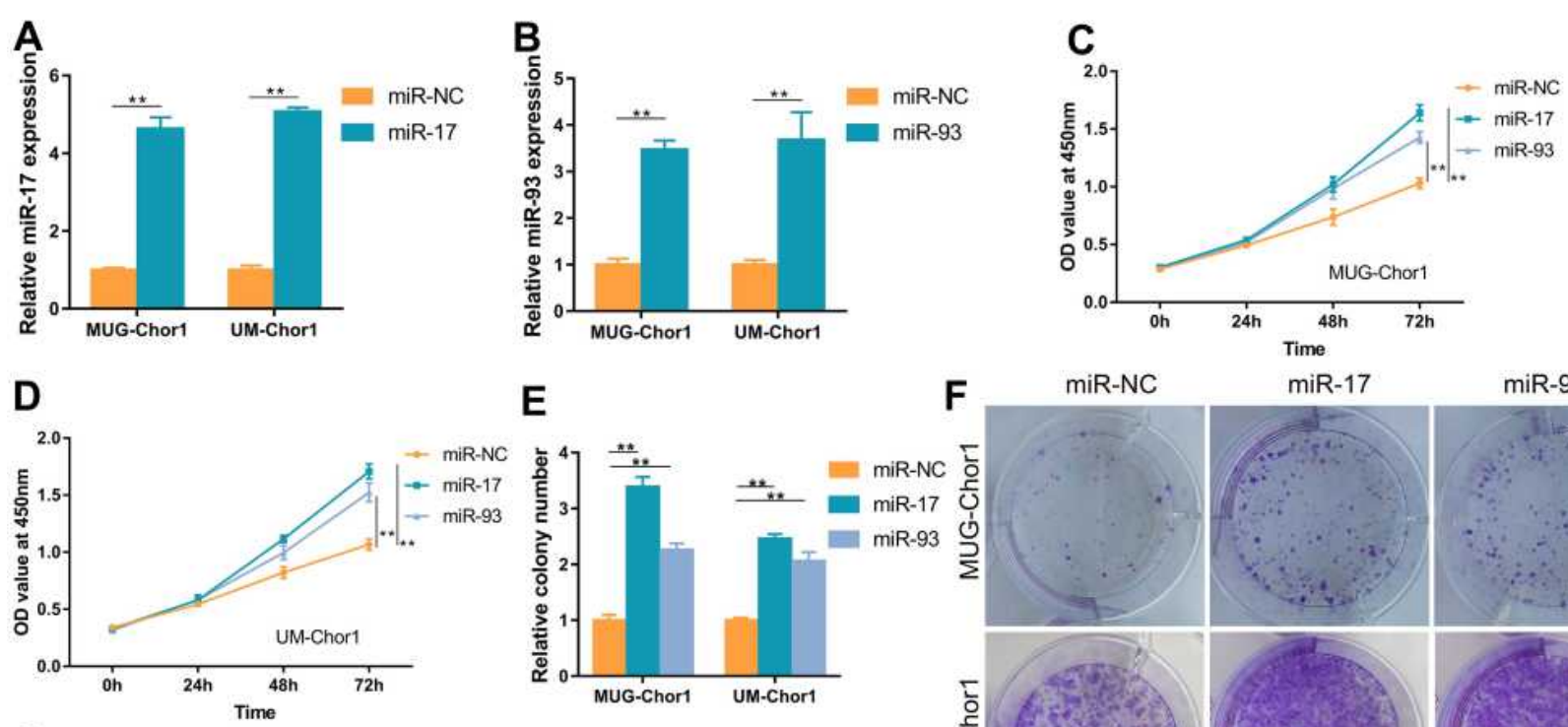

E
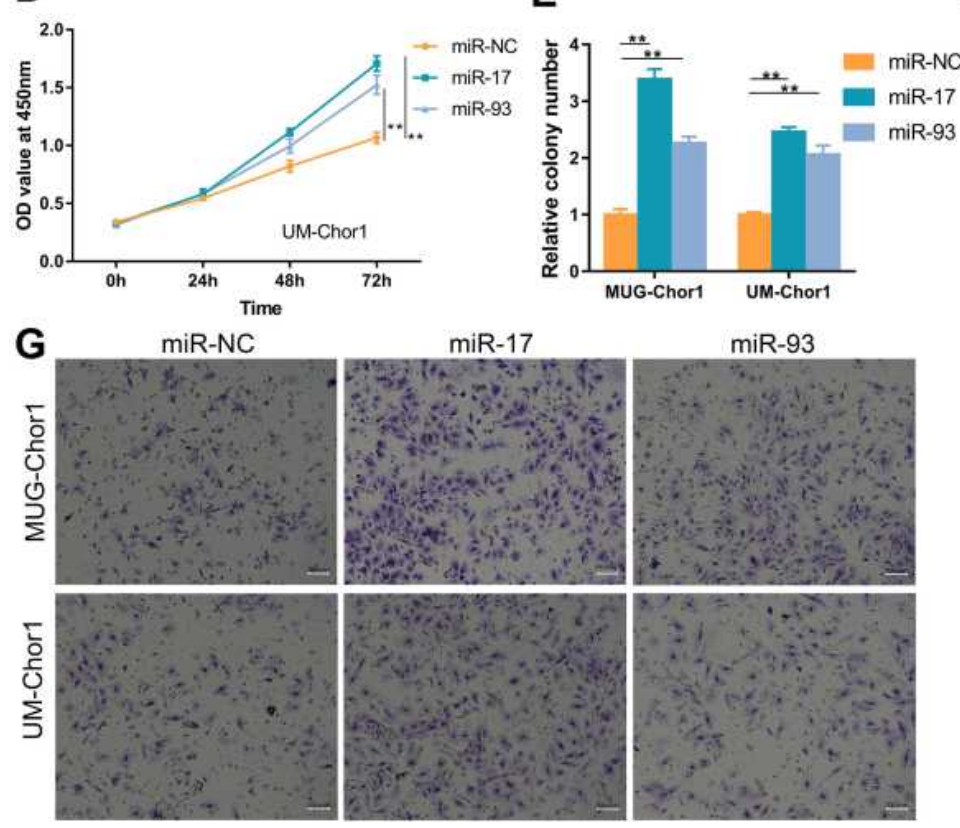

$\mathbf{F}$
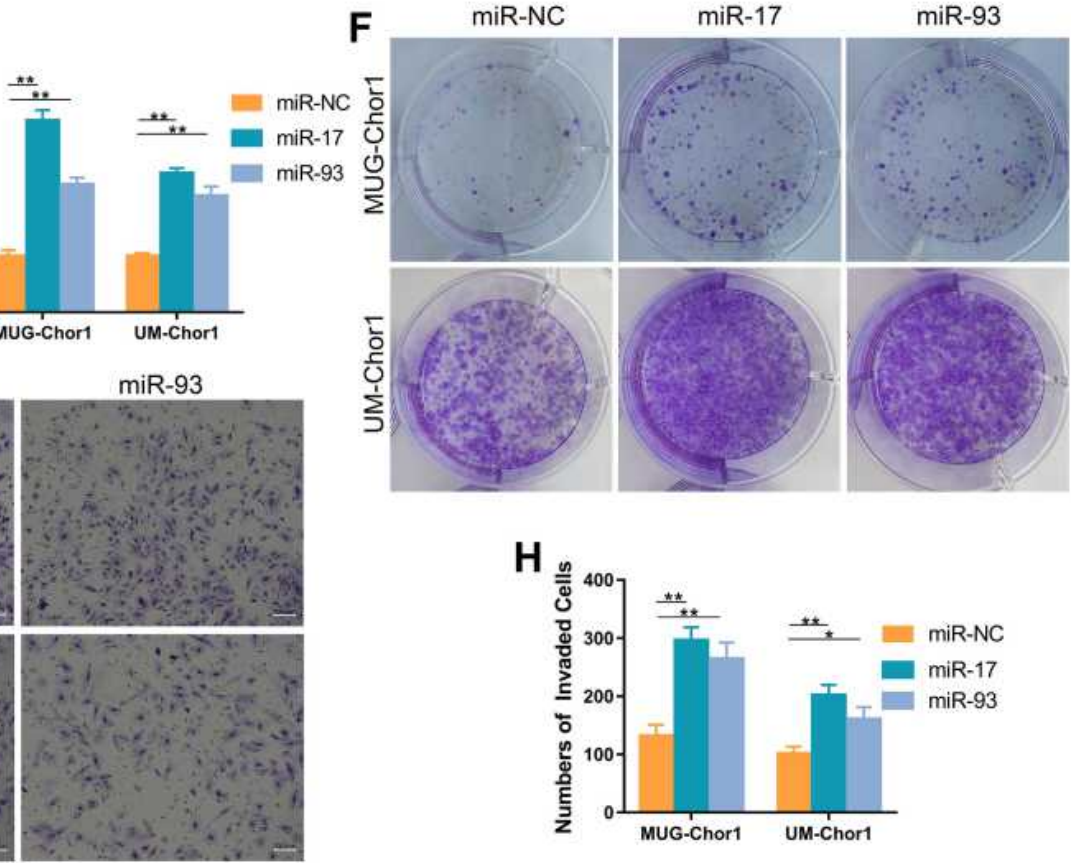

Figure 2 Overexpression of miR-I7/miR-93 promotes MUG-Chorl and UM-Chor I cell proliferation, colony formation, and invasion in vitro. (A and B) Expression of miR17/miR-93 was obviously increased by miRNA mimics. (C and D) CCK-8 assay showed that miR-17/miR-93 promotes cell proliferation compared to miR-NC. (E and F) Colony formation assay revealed that miR-17/miR-93 contribute to chordoma cell colony formation. (G and $\mathbf{H})$ Transwell invasion assay indicated the invaded ability of chordoma cells increased after miR-I7/miR-93 treated. Magnification, I00X. $* p<0.05, * * p<0.01$.

\section{Overexpression of P2I Partially Abolished the Effect of MiR-17/93 in Chordoma Cells}

We then performed the rescue assays to confirm the miR17/93-p21 axis. MUG-Chor1 and UM-Chor1 cells transfected with miR-17/93 mimics were further co-transfected with $\mathrm{p} 21$ and vector plasmid. The mRNA level and protein expression of p21 were increased after transfected with p21 over-expressed plasmid (Figure 4A and B). The effect of miR-17/93 mimics on proliferation ability (Figure 4C) and the numbers of cloning cells (Figure 4D and E) was reversed by $\mathrm{p} 21$ overexpression. Moreover, the increased invasion ability of MUG-Chor1 and UM-Chor1 cells via the miR-17/93 mimics was recovered after the cotransfection of p21 (Figure 4F and G). Taken together, our findings revealed that miR-17/93 contributed to tumor progression via directly targeting p 21 in chordoma.

\section{Discussion}

The essential role of miRNA in various diseases and tumor progression has been demonstrated in numerous studies of recent decades. ${ }^{8,13}$ In chordoma, several potential oncogenetic/tumor suppressor miRNAs have been identified and showed potent predictive performance for outcome. ${ }^{14-16,29}$ However, to our knowledge, few studies analyze the role and function of miR-17/93 in chordoma. In the current study, we for the first time character the levels of miR-17/ 93 in chordoma patients and we found a significant upregulation of miR-17/93 in chordoma. Further survival analysis showed that high expression of miR-17 or miR93 was associated with unfavorable prognosis in chordoma, while low level of p21 was correlated with the shorter OS, and sequent multivariate Cox analysis identified that miR-17 could act as an independent indicator for patient outcome prediction. Functionally, the upregulation 


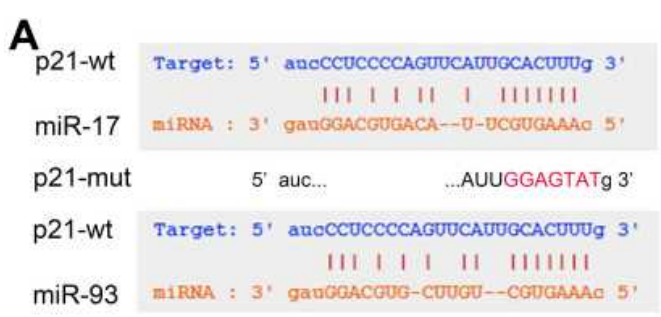

D
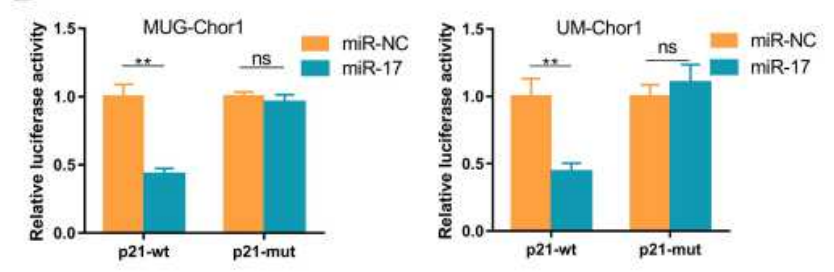

$\mathbf{F}$

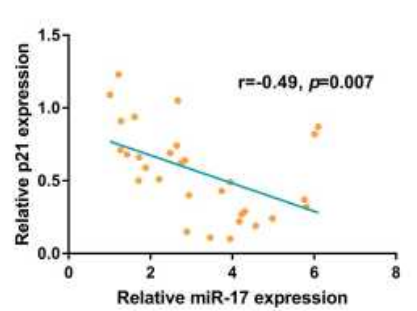

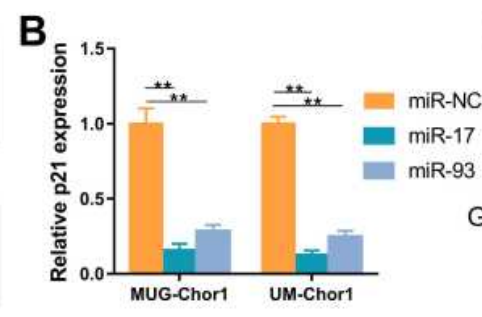

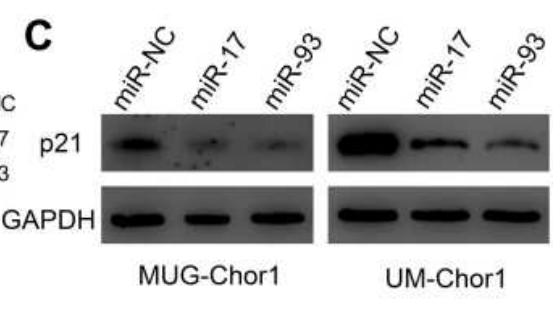

E
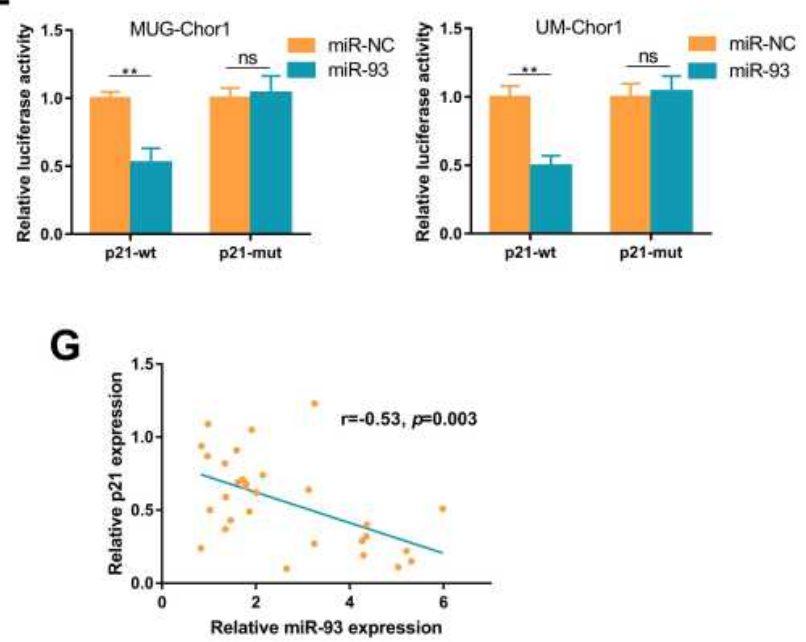

Figure 3 MiR-17 and miR-93 directly target p2I in chordoma cells. (A) Bioinformatics analysis suggests potential binding sites of miR-I7/93 to wild type (wt) p2I and subsequent construction of the mutant type (mut) p2I. (B and C) The effect of miR-I7/93 on p2I mRNA and protein expression. (D and E) Luciferase activity of chordoma cells co-transfected with either $\mathrm{p} 2 \mathrm{I}$-wt or $\mathrm{p} 2 \mathrm{I}$-mut in combination with either miR-17/93 or miR-NC. (F and G) MiR-I7/93 expression negatively correlated with p2I expression in chordoma tissues. ${ }^{* *} p<0.01$.

Abbreviation: ns, not significant.

of miR-17 and miR-93 promoted cell proliferation, colony formation, and invasion via targeting $\mathrm{p} 21$ in chordoma cells. Furthermore, the co-transfection of p21 partially reversed these cellular behaviors in chordoma cells.

The miR-17-92 cluster, one of the most well-studied miRNA clusters first identified in 2004, is located at chromosome 13q31, and aberrant expression of which has been linked to oncogenesis and tumor progression in various cancers such as stomach cancer, lymphoma, and prostate cancer. ${ }^{30}$ Increasing studies identified the potential oncogene role of miR-17, one part of the miR-17-92 cluster, in cancer progression, though some discordance exists in certain types of cancers. A high level of miR-17 was observed in pancreatic cancer, nasopharyngeal carcinoma, and Burkitt lymphoma, ${ }^{18,31,32}$ while studies involving cervical cancer and triple-negative breast cancer indicated that miR-17 could inhibit cell growth and may act as a tumor suppressor. ${ }^{33,34}$ Our results were in line with the former opinion of the oncogenic role of miR-17, demonstrated that miR-17 was up-regulated in chordoma and correlated with an adverse OS of chordoma patients. Moreover, miR-17 promotes cell growth and invasion ability in chordoma cells, indicating a potential oncogenic role of miR-17 in chordoma tumors. Exploring underlying mechanisms of the complicated roles of miR17 in cancers is highly needed, and recent studies demonstrated that miR-17 could functions via direct targeting p21 in several types of cancer cells. ${ }^{21,22}$

Similar to miR-17, miR-93 belonging to miR-106b-25 Cluster, a homolog of miR-17-92 cluster, is involved and up-regulated in various cancers, though one study involving colon cancer showed a different opinion. ${ }^{23,35}$ One study by Ma indicated the increased expression of miR93 in stomach tumors and its correlation with poor patient outcomes. Recently, Jiang reported aberrant expression of miR-93 and its promotion to granulose cell growth in polycystic ovarian syndrome. ${ }^{36}$ Interestingly, several genes including p21 are considered as targets of miR93. ${ }^{24,36}$ And a low level of cyclin kinase inhibitor p21 has been demonstrated in various tumors. ${ }^{37}$ Consistent with those studies, our data revealed that miR-93 expression was significantly increased while the level of p21 was decreased in chordoma tissues, and high miR-93 or low p21 expressions could predict the adverse outcome of 


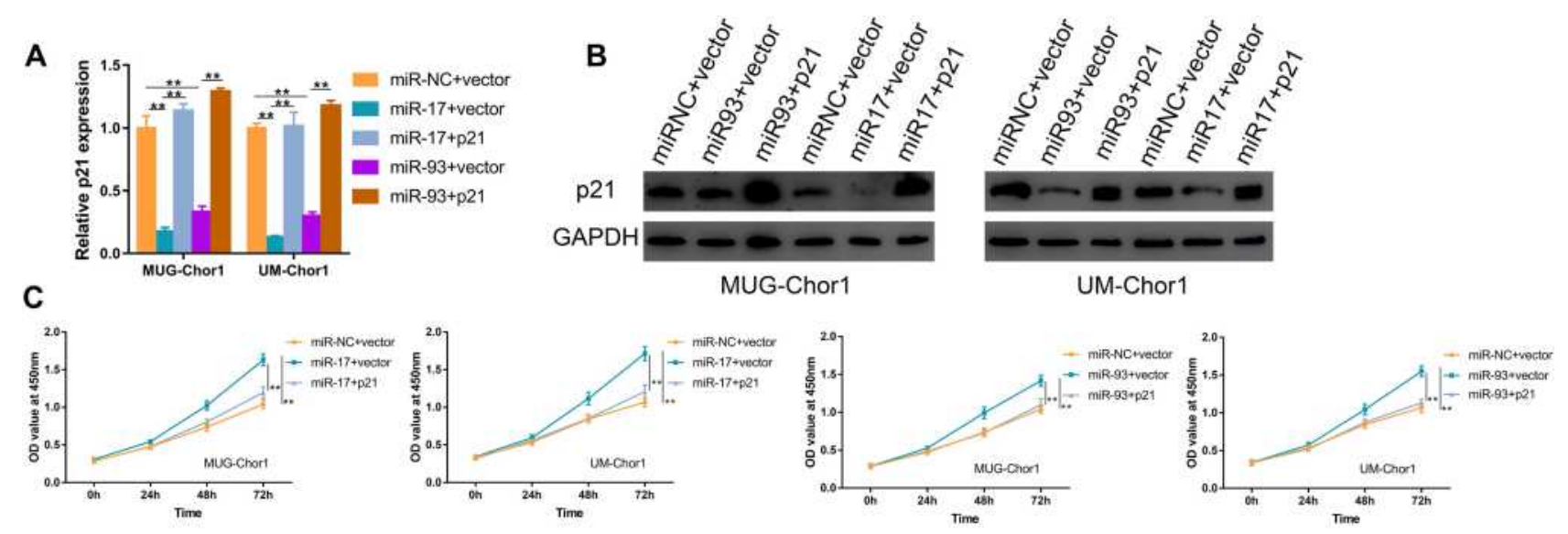

D miR-NC+vector miR-17+vector miR-17+p21 miR-93+vector miR-93+p21

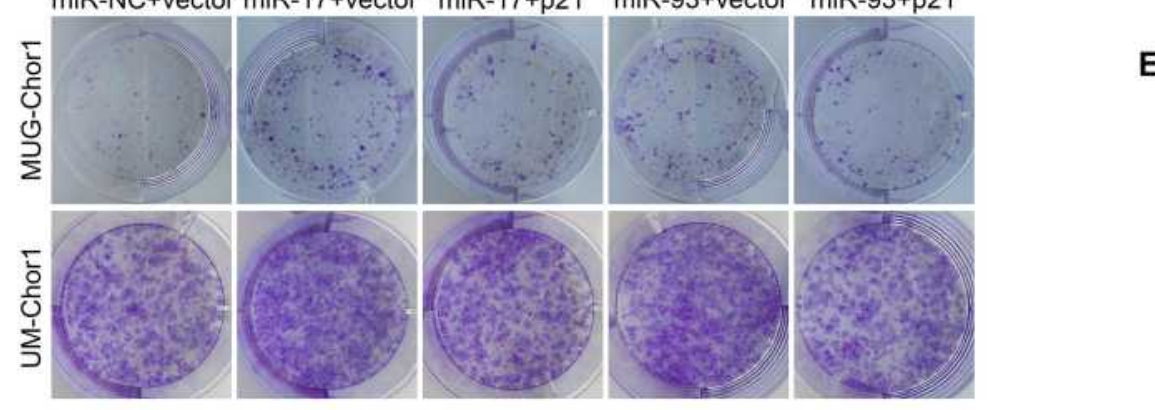

E

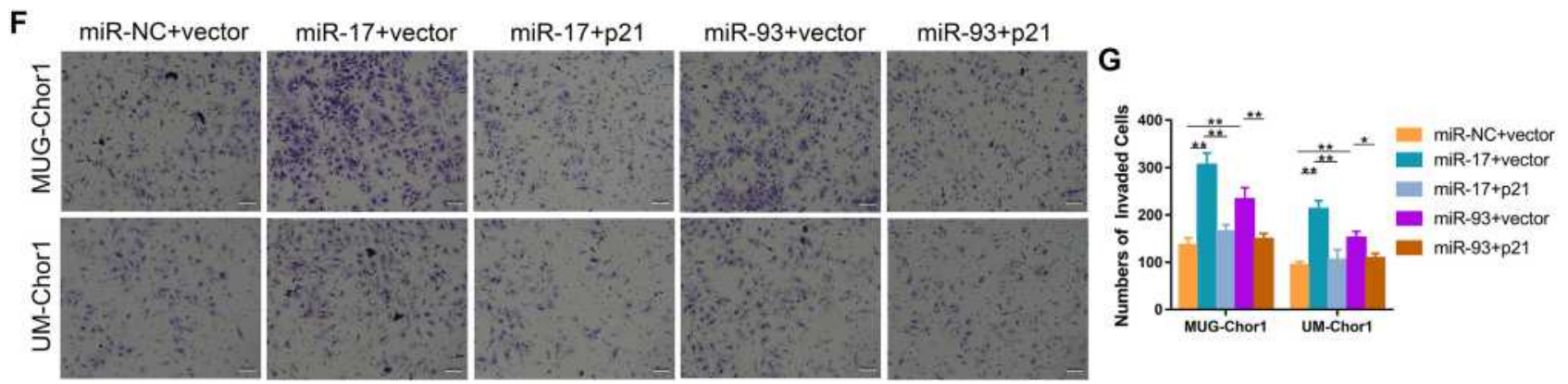

Figure 4 Overexpression of p2I counteracts the potential oncogenic effect of miR-17/93 on chordoma cells. (A and B) The qRT-PCR and Western blot were used to confirm the up-regulation of $\mathrm{p} 2 \mathrm{I}$ in chordoma cells treated with $\mathrm{p} 21$ plasmid. (C) CCK-8 assay revealed that up-regulation of p2I reversed the miR-I7/93 induced promotion of cell proliferation. (D and E) Colony formation assay showed the number of colony cells decreased after co-transfection with p2I. (F and $\mathbf{G})$ The miR-I7/93 induced promotion of chordoma cell invasion was rescued after co-transfection with $p 21$. Magnification, $100 X . * p<0.05, * * p<0.0$ I.

chordoma patients. Functional studies further confirmed the potential oncogenic role of miR-93 in chordoma cells. Importantly, our results revealed that miR-17 and miR-93 functioned via directly targeting p21 in chordoma, and identified the possible impact of the miR-17/93-p21 axis on chordoma progression.

\section{Conclusions}

Our results demonstrate that upregulation of miR-17/93 and decreased p21 expression is associated with poor outcomes, and miR-17 is an independent indicator of overall survival in chordoma. Our study reveals that targeting the
miR-17/93-p21 axis may be a potential therapy for chordoma patients. Further large-scale studies are wanted to validate our findings.

\section{Abbreviations}

miRNAs, micorRNAs; OS, overall survival; qRT-PCR, quantitative real-time polymerase chain reaction.

\section{Data Sharing Statement}

All data generated or analyzed during this study are included in this published article and its Supplementary Information Files. 


\section{Ethics Approval and Informed Consent}

The current study was approved by the ethics committee of Tangshan People's Hospital and carried out according to the Declaration of Helsinki. And informed consent was obtained from all participants.

\section{Author Contributions}

All authors made a significant contribution to the work reported, whether that is in the conception, study design, execution, acquisition of data, analysis, and interpretation, or in all these areas; took part in drafting, revising, or critically reviewing the article; gave final approval of the version to be published; have agreed on the journal to which the article has been submitted; and agree to be accountable for all aspects of the work.

\section{Funding}

This study was supported by the Natural Science Foundation of Hebei Province (grant number H2020105017).

\section{Disclosure}

The authors declare that they have no competing interests.

\section{References}

1. Salisbury JR, Deverell MH, Cookson MJ, Whimster WF. Threedimensional reconstruction of human embryonic notochords: clue to the pathogenesis of chordoma. J Pathol. 1993;171(1):59-62. doi:10.1002/path.1711710112

2. Walcott BP, Nahed BV, Mohyeldin A, Coumans J-V, Kahle KT, Ferreira MJ. Chordoma: current concepts, management, and future directions. Lancet Oncol. 2012;13(2):e69-e76. doi:10.1016/S14702045(11)70337-0

3. Smoll NR, Gautschi OP, Radovanovic I, Schaller K, Weber DC. Incidence and relative survival of chordomas: the standardized mortality ratio and the impact of chordomas on a population. Cancer. 2013;119(11):2029-2037. doi:10.1002/cncr.28032

4. Stacchiotti S, Casali PG, Vullo SL, et al. Chordoma of the mobile spine and sacrum: a retrospective analysis of a series of patients surgically treated at two referral centers. Ann Surg Oncol. 2010;17 (1):211-219. doi:10.1245/s10434-009-0740-x

5. Di Maio S, Temkin N, Ramanathan D, Sekhar LN. Current comprehensive management of cranial base chordomas: 10-year meta-analysis of observational studies. $J$ Neurosurg. 2011;115(6):1094-1105. doi:10.3171/2011.7.JNS11355

6. Booton R, Lindsay MA. Emerging role of MicroRNAs and long noncoding RNAs in respiratory disease. Chest. 2014;146(1):193-204. doi:10.1378/chest.13-2736

7. Griffiths-Jones S, Grocock RJ, Van Dongen S, Bateman A, Enright AJ. miRBase: microRNA sequences, targets and gene nomenclature. Nucleic Acids Res. 2006;34(suppl_1):D140-D144. doi:10.1093/nar/ gkj112
8. Mendell JT, Olson EN. MicroRNAs in stress signaling and human disease. Cell. 2012;148(6):1172-1187. doi:10.1016/j. cell.2012.02.005

9. Marcucci G, Mrózek K, Radmacher MD, Garzon R, Bloomfield CD. The prognostic and functional role of microRNAs in acute myeloid leukemia. Blood. 2011;117(4):1121-1129.

10. Ishiguro $\mathrm{H}$, Kimura $\mathrm{M}$, Takeyama $\mathrm{H}$. Role of microRNAs in gastric cancer. World J Gastroenterol. 2014;20(19):5694.

11. Huang Z, Huang S, Wang Q, et al. MicroRNA-95 promotes cell proliferation and targets sorting Nexin 1 in human colorectal carcinoma. Cancer Res. 2011;71(7):2582-2589. doi:10.1158/00085472.CAN-10-3032

12. Porkka KP, Pfeiffer MJ, Waltering KK, Vessella RL, Tammela TL, Visakorpi T. MicroRNA expression profiling in prostate cancer. Cancer Res. 2007;67(13):6130-6135. doi:10.1158/0008-5472.CAN07-0533

13. Garzon R, Calin GA, Croce CM. MicroRNAs in cancer. Annu Rev Med. 2009;60:167-179. doi:10.1146/annurev.med.59.053006.104707

14. Zou M-X, Huang W, Wang X-B, et al. Reduced expression of miRNA-1237-3p associated with poor survival of spinal chordoma patients. Eur Spine J. 2015;24(8):1738-1746. doi:10.1007/s00586015-3927-9

15. Duan Z, Shen J, Yang X, et al. Prognostic significance of miRNA-1 (miR-1) expression in patients with chordoma. J Orthopaed Res. 2014;32(5):695-701. doi:10.1002/jor.22589

16. Osaka E, Kelly AD, Spentzos D, et al. MicroRNA-155 expression is independently predictive of outcome in chordoma. Oncotarget. 2015;6(11):9125. doi:10.18632/oncotarget.3273

17. Xi Y, Li J, Zhang P, et al. Upregulation of miRNA-17 and miRNA-19 is associated with unfavorable prognosis in patients with T-cell lymphoblastic lymphoma. Exp Mol Pathol. 2015;99(2):297-302. doi:10.1016/j.yexmp.2015.07.012

18. Robaina MC, Faccion RS, Mazzoccoli L, et al. miR-17-92 cluster components analysis in Burkitt lymphoma: overexpression of miR-17 is associated with poor prognosis. Ann Hematol. 2016;95 (6):881-891. doi:10.1007/s00277-016-2653-7

19. Ji C, Liu H, Yin Q, Li H, Gao H. miR-93 enhances hepatocellular carcinoma invasion and metastasis by EMT via targeting PDCD4. Biotechnol Lett. 2017;39(11):1621-1629. doi:10.1007/s10529-0172403-5

20. Ma D-H, Li B-S, Liu -J-J, et al. miR-93-5p/IFNAR1 axis promotes gastric cancer metastasis through activating the STAT3 signaling pathway. Cancer Lett. 2017;408:23-32. doi:10.1016/j. canlet.2017.08.017

21. Yang C, Yuan W, Yang X, et al. Circular RNA circ-ITCH inhibits bladder cancer progression by sponging miR-17/miR-224 and regulating p21, PTEN expression. Mol Cancer. 2018;17(1):19.

22. Wang Z, Ji F. Downregulation of microRNA-17-5p inhibits drug resistance of gastric cancer cells partially through targeting p21. Oncol Lett. 2018;15(4):4585-4591. doi:10.3892/ol.2018.7822

23. Zhang Y, Xu Z. miR-93 enhances cell proliferation by directly targeting CDKN1A in nasopharyngeal carcinoma. Oncol Lett. 2018;15(2):1723-1727.

24. Choi JY, Shin HJ, Bae IH. miR-93-5p suppresses cellular senescence by directly targeting Bcl-w and p21. Biochem Biophys Res Commun. 2018;505(4):1134-1140. doi:10.1016/j.bbrc.2018.10.010

25. Ma Y, Zhu B, Liu X, et al. iASPP overexpression is associated with clinical outcome in spinal chordoma and influences cellular proliferation, invasion, and sensitivity to cisplatin in vitro. Oncotarget. 2017;8 (40):68365-68380. doi:10.18632/oncotarget.20190

26. Zhang H, Yang K, Ren T, Huang Y, Tang X, Guo W. miR-16-5p inhibits chordoma cell proliferation, invasion and metastasis by targeting Smad3. Cell Death Dis. 2018;9(6):680.

27. Liu L, Wang T, Yang X, et al. MTNR1B loss promotes chordoma recurrence by abrogating melatonin-mediated beta-catenin signaling repression. J Pineal Res. 2019; 12588. 
28. Li JH, Liu S, Zhou H, Qu LH, Yang JH. starBase v2.0: decoding miRNA-ceRNA, miRNA-ncRNA and protein-RNA interaction networks from large-scale CLIP-Seq data. Nucleic Acids Res. 2014;42 (Database issue):D92-97. doi:10.1093/nar/gkt1248

29. Duan Z, Choy E, Nielsen GP, et al. Differential expression of microRNA (miRNA) in chordoma reveals a role for miRNA-1 in Met expression. J Orthopaed Res. 2010;28(6):746-752. doi:10.1002/ jor.21055

30. Bonauer A, Dimmeler S. The microRNA-17 92 cluster: still a miRacle? Cell Cycle. 2009;8(23):3866-3873. doi:10.4161/ cc.8.23.9994

31. Yu J, Ohuchida K, Mizumoto K, Fujita H, Nakata K, Tanaka M. MicroRNA miR-17-5p is overexpressed in pancreatic cancer, associated with a poor prognosis, and involved in cancer cell proliferation and invasion. Cancer Biol Ther. 2010;10(8):748-757.

32. Chen H, Chen G, Chen Y, et al. MicroRNA deregulation and pathway alterations in nasopharyngeal carcinoma. Br J Cancer. 2009;100 (6):1002-1011. doi:10.1038/sj.bjc.6604948
33. Li J, Lai Y, Ma J, et al. miR-17-5p suppresses cell proliferation and invasion by targeting ETV1 in triple-negative breast cancer. $B M C$ Cancer. 2017;17(1):745.

34. Wei Q, Li YX, Liu M, Li X, Tang H. MiR-17-5p targets TP53INP1 and regulates cell proliferation and apoptosis of cervical cancer cells. IUBMB Life. 2012;64(8):697-704. doi:10.1002/iub.1051

35. Tang Q, Zou Z, Zou C, et al. MicroRNA-93 suppress colorectal cancer development via Wnt/ $\beta$-catenin pathway downregulating. Tumor Biol. 2015;36(3):1701-1710. doi:10.1007/s13277-014-2771-6

36. Jiang L, Huang J, Li L, et al. MicroRNA-93 promotes ovarian granulosa cells proliferation through targeting CDKN1A in polycystic ovarian syndrome. J Clin Endocrinol Metab. 2015;100(5):E729E738.

37. Abbas T, Dutta A. p21 in cancer: intricate networks and multiple activities. Nat Rev Cancer. 2009;9(6):400-414. doi:10.1038/nrc2657

\section{Publish your work in this journal}

OncoTargets and Therapy is an international, peer-reviewed, open access journal focusing on the pathological basis of all cancers, potential targets for therapy and treatment protocols employed to improve the management of cancer patients. The journal also focuses on the impact of management programs and new therapeutic agents and protocols on patient perspectives such as quality of life, adherence and satisfaction. The manuscript management system is completely online and includes a very quick and fair peer-review system, which is all easy to use. Visit http://www.dovepress.com/ testimonials.php to read real quotes from published authors. 